\title{
Un enfoque etnomatemático de la modelación a través de la Etnomodelación
}

\author{
Milton Rosa \\ Universidad Federal de Ouro Preto \\ milton.rosa@ufop.edu.br \\ Daniel Clark Orey \\ Universidad Federal de Ouro Preto \\ oreydeema@gmail.com
}

Recibido: 9-febrero-2018 / Aceptado: 31 - julio - 2018

\section{Resumen}

Es importante buscar enfoques metodológicos alternativos, mientras las prácticas matemáticas occidentales sean aceptadas a nivel mundial, para registrar formas históricas de ideas y procedimientos matemáticos que se dan en diferentes contextos culturales. Un enfoque metodológico alternativo es el de la etnomodelación, que consideramos como una aplicación práctica de la etnomatemática que agrega la perspectiva cultural a conceptos de modelación matemática. En este contexto, sofisticadas ideas y prácticas matemáticas, que incluyen principios geométricos en trabajo artesanal, conceptos arquitectónicos y prácticas, son encontrados en actividades y artefactos de muchas culturas locales y globales. Estos conceptos están relacionados con las relaciones numéricas que se encuentran en la medición, el cálculo, los juegos, la adivinación, la navegación, la astronomía, el modelado y en una amplia variedad de otros procedimientos matemáticos, tanto como en artefactos culturales a través del desarrollo de la etnomodelación.

Palabras clave: acción pedagógica, etnomatemáticas, etnomodelación, etnomodelos, modelación. 


\begin{abstract}
It is important to seek for alternative methodological approaches, while western mathematical practices are accepted worldwide to record historical forms of mathematical ideas and procedures that occur in different cultural contexts. An alternative methodological approach is ethnomodelling, which we consider as the practical application of ethnomathematics that adds the cultural perspective to the concepts of mathematical modelling. In this context, sophisticated mathematical ideas and practices, including geometric principles in craftwork, architectural concepts and practices, are found in activities and artifacts of many local and global cultures. These concepts are related to numerical relationships found in measurement, calculation, games, divination, navigation, astronomy, modelling and in a wide variety of other mathematical procedures, as well as in cultural artifacts, through the development of ethnomodelling.
\end{abstract}

Keywords: pedagogical action, ethnomathematics, ethnomodelling, ethnomodels, modelling. 


\section{Consideraciones iniciales}

$\mathrm{L}$

as matemáticas son frecuentemente presentadas como una asignatura universal que posee un lenguaje propio. Sin embargo, las diferencias culturales, lingüísticas y procedimentales para la resolución de los problemas matemáticos enfrentados por estudiantes en las escuelas, son una cuestión educacional que necesita ser investigada. Si estas diferencias no son discutidas, serán adicionadas a las dificultades que los estudiantes encuentren en clase.

De acuerdo con Rosa y Orey (2006), la vida cotidiana de los miembros de diferentes grupos culturales puede ser percibida como si fuera representación de la propia realidad, que está generada, vía inferencias, con la utilización de representaciones mentales a través de la modelación en una perspectiva de las etnomatemáticas.

En ese sentido, la utilización de las ideas, procedimientos y prácticas matemáticas que están presentes en el día a día de tales miembros tiene por objetivo la ampliación y el perfeccionamiento de su conocimiento matemático, pues busca el fortalecimiento de la identidad cultural de los individuos, como seres autónomos y capaces.

Siendo así, es importante que los individuos desarrollen sus propias prácticas matemáticas. Sin embargo, es fundamental que también tengan una comprensión de la institución sociopedagógica de la matemática por medio de acciones pedagógicas que fomenten la conexión de las prácticas matemáticas presentes en la comunidad con los contenidos matemáticos enseñados en las escuelas, en una reinterpretación del currículo matemático escolar (D’Ambrosio, 1990).

Es necesario buscar enfoques metodológicos alternativos, mientras las prácticas matemáticas occidentales sean aceptadas a nivel mundial, para registrar formas históricas de ideas matemáticas que se dan en diferentes circunstancias culturales. En este contexto, un enfoque metodológico alternativo es el de la etnomodelación, que consideramos como una aplicación práctica de la etnomatemática, que agrega la perspectiva cultural a conceptos de modelación matemática.

La etnomodelación debe proporcionar a los estudiantes una acción pedagógica que conecte esas prácticas matemáticas a las proporcionadas por la adquisición de los conocimientos de la matemática académica. De esta manera, Rosa y Orey (2017) sostienen que la propuesta de la etnomodelación puede ser interpretada como una metodología que permita reconocer y presentar las matemáticas presentes en el día a día de los alumnos en situaciones didácticas motivadoras.

\section{Delineando un camino para la acción pedagógica de la etnomodelación}

Para que podamos delinear un camino para la acción pedagógica de la etnomodelación, existe la necesidad de:

1) Realizar un trabajo de investigación de campo para que podamos entender cuáles ideas, procedimientos o prácticas matemáticas, presentes en la comunidad, pueden ser considerados como objetos de estudios pedagógicos.

2) Determinar cuál propuesta educacional debe ser considerada para la elaboración del currículo matemático escolar. 
Ante estas pretensiones comprendemos que el proceso pedagógico de la etnomodelación está relacionado con los abordajes propuestos por Eglash (2002) que delinearán esta acción pedagógica, y que están relacionados con los sistemas de conocimiento profundamente vinculados al día a día de los miembros de cada grupo cultural y que pueden ser matematizados y traducidos al lenguaje de las matemáticas académicas.

Así, entendemos que matematizar determinadas ideas, procedimientos y prácticas matemáticas presentes diariamente en diferentes grupos culturales es trabajar con las etnomatemáticas (Rosa y Orey, 2010). Entonces, existe la necesidad de destacar el proceso de la modelación por medio de ejemplos de la utilización de técnicas matematizadoras que sean desarrolladas por los miembros de diferentes grupos culturales y también su encuentro natural con las etnomatemáticas (Bassanezi, 2002).

Por ejemplo, un grupo de estudiantes participando en un curso de especialización en Brasil, procuró comprender, entender, y saber cuáles eran las matemáticas utilizadas por don Joaquín, en Ijuí, Rio Grande del Sur, que producía vinos y construía sus propios toneles, utilizando ideas, procedimientos y prácticas matemáticas que le fueron transmitidos por sus antepasados italianos. La figura 1 muestra la matematización del tonel de vino.

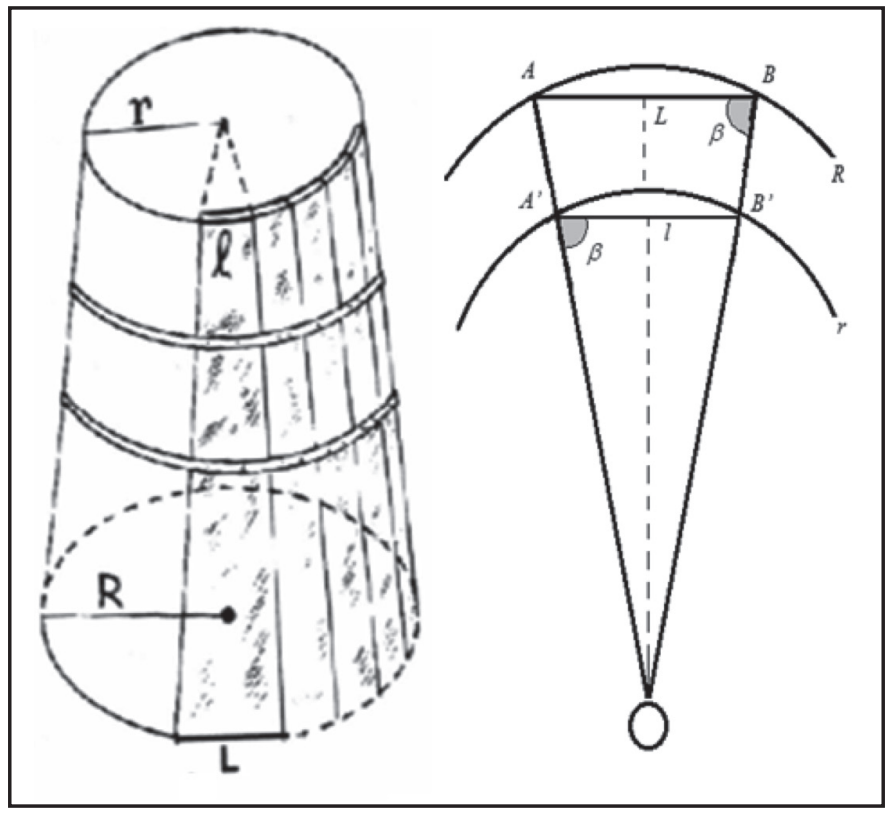

Figura 1. Matematización del tonel de vino.

Fuente: Bassanezi (2002)

En otro estudio, Ríos (2000) también procuró entender y comprender el proceso mental de idealización de ponchos, que son vestimentas utilizadas como abrigo 
o sobretodo, y aguayos, que son vestimentas utilizadas como mantillas, y que son confeccionados por las campesinas bolivianas. La figura 2 muestra los ponchos y aguayos bolivianos.

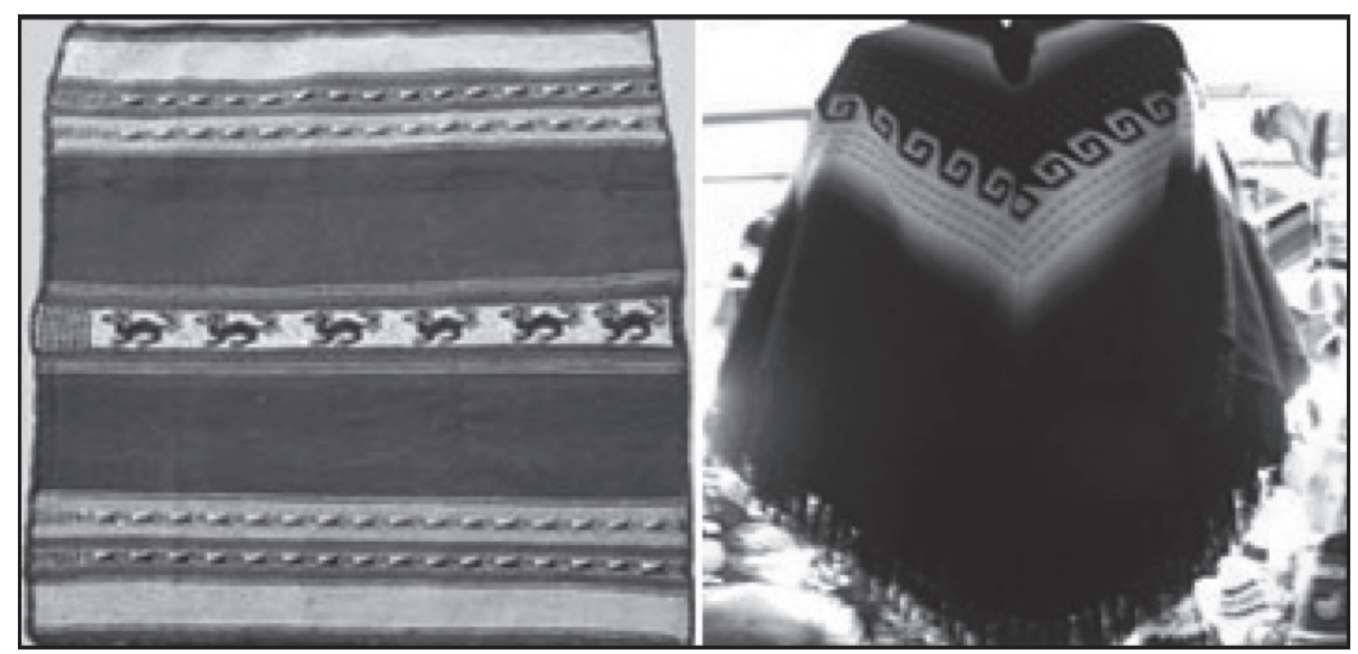

Figura 2. Ponchos y aguayos bolivianos.

Fuente: Rosa y Orey (2017)

En esta investigación, Ríos (2000) describió las prácticas matematizadoras que se utilizan en la confección de estos tipos de vestimentas y observó que, durante este trabajo, las campesinas están constantemente evaluando y analizando los resultados, alterándolos en caso de que el modelo obtenido no esté de acuerdo con las representaciones mentales que fueron previamente concebidas.

De forma similar, Knijnik (1993) también utilizó un abordaje etnomatemático para matematizar el conocimiento de los trabajadores del Movimiento Sin Tierra, en Brasil, al evaluar áreas de tierras y calcular el volumen de troncos de árboles. Para desarrollar este proceso de cubicación, esta investigadora elaboró una traducción de este conocimiento para el lenguaje matemático demostrando su valor y su utilización para la práctica pedagógica.

En esta misma línea de estudio, Gerdes (1993) matematizó los dibujos en arena, denominados sona, que son elaborados por los nativos de Angola y Zambia, legitimando y valorando el reconocimiento de esta práctica cultural, traduciendo estos conocimientos para el currículo escolar con la utilización de la matemática académica. La figura 3 muestra un dibujo sona. 


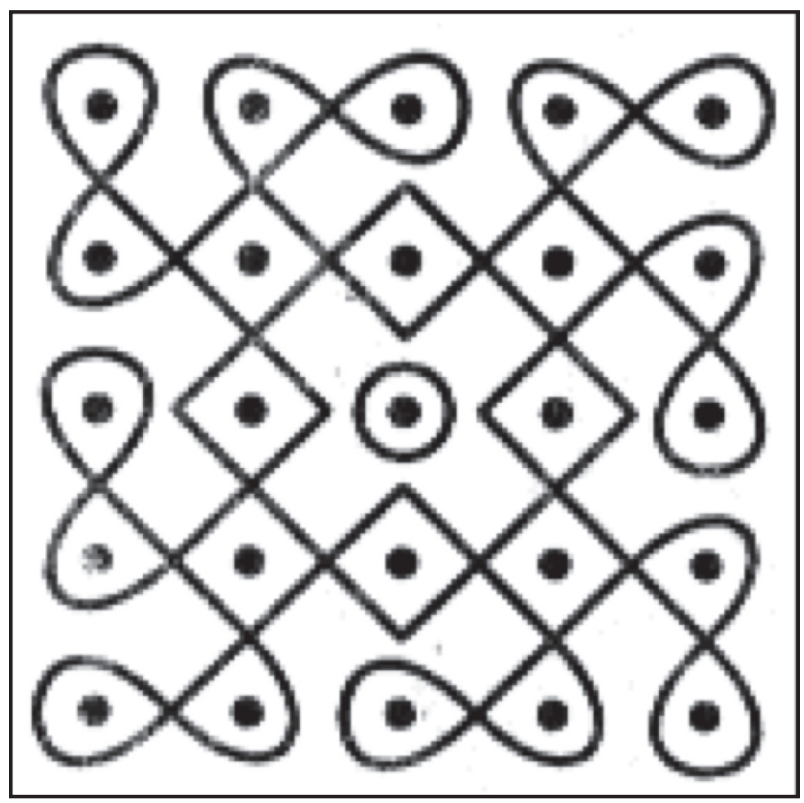

Figura 3. Dibujo en arena, zona.

Fuente: Rosa y Orey (2017)

Estos estudios revelan que la matematización de la realidad, elaborada por miembros de diferentes grupos culturales se ve como representación de la propia realidad que se genera, vía inferencias, con la utilización de representaciones mentales basadas en los conocimientos matemáticos locales.

Es importante resaltar que estas investigaciones también demuestran que, de acuerdo con Monteiro y Pompeu Jr. (2003), la propuesta etnomatemática puede ser interpretada como un programa que permite reconocer y presentar las matemáticas, presentes en el día a día de los estudiantes, en situaciones didácticas motivadoras.

\section{Etnomatemáticas + Modelación $=$ Etnomodelación}

Partiendo del principio de que la matematización es una de las etapas más importantes de la modelación, pues traduce las situaciones problema para el lenguaje matemático académico, entendemos que la modelación es una de las posibles propuestas para iniciar la acción pedagógica del programa etnomatemáticas por medio de la etnomodelación.

En este sentido, la utilización de las etnomatemáticas, que están presentes en el día a día de los miembros de los grupos culturales, tiene por objetivo la ampliación y el perfeccionamiento de su conocimiento matemático, pues busca el fortalecimiento de la identidad cultural de los individuos, como seres autónomos y capaces (Rosa y Orey, 2003).

Del mismo modo, la modelación es una de las posibles estrategias de enseñanza que puede hacer posible la aproximación y la relación entre el hacer y saber escolar 
y el día a día (Monteiro, 2004). Este aspecto también considera el uso pedagógico sobre los modos por los cuales podamos conectar la matemática formal al contexto cultural, en el currículo matemático (Orey, 2000).

Consecuentemente, la modelación proporciona al educando la utilización de instrumentos comunicativos, analíticos y materiales que son esenciales para el ejercicio de los derechos y deberes, necesarios para la práctica de la ciudadanía y la lectura crítica y reflectante de los fenómenos que ocurren en el mundo globalizado (D’Ambrosio, 2000).

En ese contexto, las etnomatemáticas ofrecen una visión más amplia de las matemáticas, pues abarcan las ideas, nociones, procedimientos, procesos, métodos y prácticas arraigadas en entornos culturales distintos. Este aspecto conduce a una mayor evidencia de los procesos cognitivos, capacidades de aprendizaje y actitudes que los procesos de aprendizaje directo que ocurren en las aulas (Rosa, 2010).

Además, mediante la reflexión sobre las dimensiones sociales y políticas de la etnomodelación, otro aspecto importante de este campo de estudio es la posibilidad de la aplicación de enfoques innovadores para el desarrollo de una sociedad dinámica y glocalizada. ${ }^{1}$

\section{Etnomodelación}

Para Rosa y Orey (2017), las prácticas matemáticas que se refieren a las relaciones numéricas encontradas en la medición, la clasificación, el cálculo, los juegos, la adivinación, la navegación, la astronomía, la modelación y, también, en una amplia variedad de otros procedimientos matemáticos son empleadas en la producción de los artefactos culturales por los miembros de grupos culturales distintos.

Ese contexto permite el desarrollo de una definición de etnomodelación como la traducción de las ideas locales matemáticas, los procedimientos y las prácticas en las que el prefijo etno- se refiere al conocimiento matemático específico desarrollado por los miembros de grupos culturales distintos (Rosa y Orey, 2017). Por lo tanto, es necesario comenzar con el contexto social, la realidad y los intereses de los estudiantes y no mediante la aplicación de un conjunto de valores externos a los que se ven obligados (D’Ambrosio, 1990).

En tal sentido, Rosa y Orey (2012) afirman que el aspecto principal de la etnomodelación es resolver los problemas presentes en su vida cotidiana, así como promover la creación de una sencilla comprensión de los sistemas matemáticos alternativos y, también, para que los estudiantes puedan entender mejor y más acerca de la importancia y el papel de las matemáticas en su sociedad y contexto.

En esta dirección, con la utilización de la etnomodelación como herramienta en la acción pedagógica del programa Etnomatemática, los estudiantes han demostra-

1 Para Rosa y Orey (2017), la glocalización (global + local) es un abordaje dialógico que considera la interacción entre los conocimientos matemáticos locales (desde dentro/émicos/insiders) y globales (desde fuera/éticos/outsiders). Este enfoque también está relacionado con la aceleración e intensificación de la interacción e integración entre los miembros de grupos culturales distintos que componen la sociedad. 
do que aprenden cómo encontrar y trabajar con situaciones auténticas y problemas de la vida real (Rosa \& Orey, 2010). De esta manera, la etnomodelación se configura como el elemento esencial en el ámbito de la antropología cultural, la etnomatemática y la modelación matemática. La figura 4 muestra la intersección entre estos tres campos de estudio.

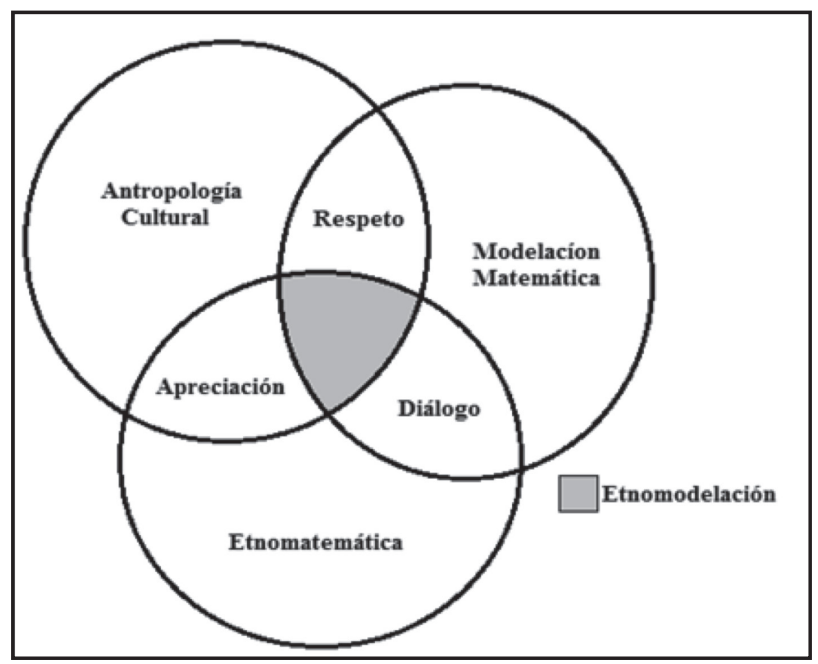

Figura 4. Etnomatemática como intersección entre tres campos de estudio.

Fuente: Rosa y Orey (2012)

De acuerdo con la figura 4, la traducción es la descripción de los procesos de modelación de sistemas locales (culturales), los cuales pueden tener una representación matemática en la cultura occidental y viceversa. Por ejemplo, para Rosa y Orey (2003), la etnomatemática hace uso de la modelación a fin de establecer relaciones entre los marcos conceptuales locales y las matemáticas incluidas en los diseños globales. Y, según su punto de vista, esto no es diferente a la traducción de palabras, términos, ideas y conceptos del español al inglés o al portugués.

\section{Etnomodelación: conocimientos émico y ético}

El conocimiento matemático puede ser visto como un resultado de origen émico (desde dentro) más que ético (desde fuera) (Eglash, 2006). Por ejemplo, Lewis (2018) sostiene que la perspectiva parece razonable, ya que las etnomatemáticas (émico) aplican la modelación (ético) para establecer relaciones en el marco conceptual entre el conocimiento local (émico) y el conocimiento matemático académico (ético). 


\section{Conocimiento matemático émico}

El conocimiento matemático émico está relacionado con las cuentas, las descripciones y el análisis y las prácticas matemáticas que son expresadas en términos de las categorías y esquemas conceptuales que se consideran significativos y apropiados por los miembros de diferentes grupos culturales (Rosa y Orey, 2012).

Este conocimiento matemático está de acuerdo con las percepciones e interpretaciones consideradas apropiadas por la cultura desde dentro. Consecuentemente, este conocimiento está relacionado con los saberes y haceres matemáticos desarrollados por los miembros de estos grupos desde dentro de la cultura por medio de una visión interna de este conocimiento (Rosa y Orey, 2017). La figura 5 muestra un ejemplo de conocimiento matemático émico.

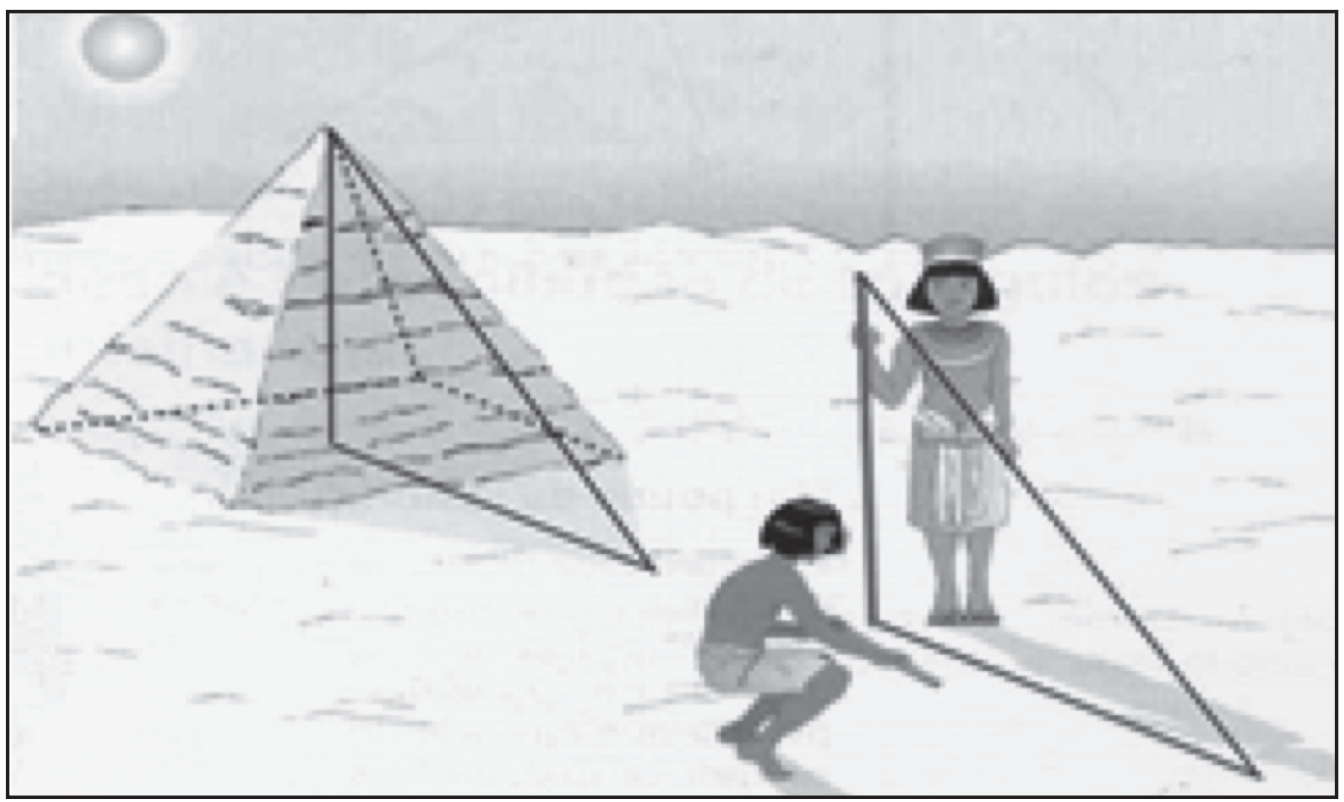

Figura 5. Conocimiento matemático émico.

Fuente: Rosa y Orey (2017)

Es importante resaltar que, para Rosa y Orey (2017), la validación del conocimiento émico trae consigo una cuestión de consenso de la población local, que debe estar de acuerdo con que estas construcciones, coinciden con las percepciones compartidas que retratan las características de su cultura.

\section{Un ejemplo de conocimiento émico}

La escultura de marfil mangbetu de Zaire (Babbitt et al., 2011) tiene una técnica de construcción que usa un ángulo de 45 grados y las propiedades de escalamiento de la talla de marfil pueden revelar su estructura subyacente que tiene tres características geométricas interesantes: 
En primer lugar, cada cabeza es mayor que la de abajo, y mira en la dirección opuesta; en segundo lugar, cada cabeza está enmarcada por dos líneas, una formada por la mandíbula y otra formada por el pelo, que se intersecan aproximadamente a 90 grados y, en tercer lugar, hay una asimetría: el lado izquierdo muestra un ángulo de cerca de 20 grados con la vertical (Eglash, 2011).

Esta secuencia de cuadrados que se van encogiendo puede ser construida por un proceso iterativo, bisecando la longitud del lado de un cuadrado para crear la longitud del lado del cuadrado siguiente. En esta escultura, el lado izquierdo está a unos 20 grados de la vertical. En la estructura iterativa de cuadrados, el lado izquierdo está aproximadamente a 18 grados de la vertical (Eglash, 2011). La figura 6 muestra una escultura de marfil mangbetu de Zaire y la secuencia de cuadrados.

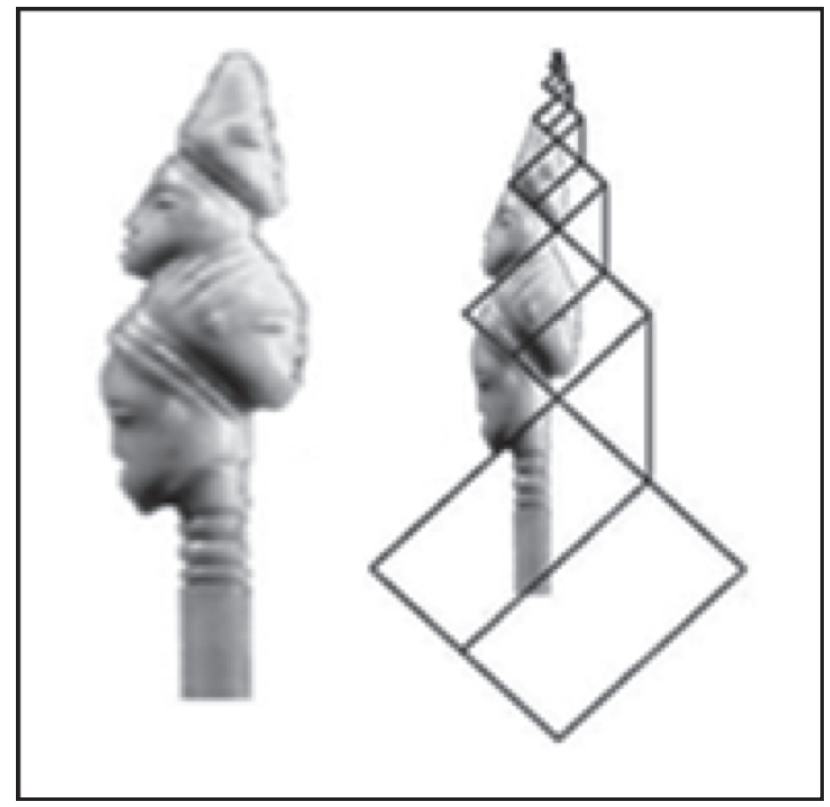

Figura 6. Escultura de marfil mangbetu y la secuencia de cuadrados.

Fuente: Eglash (2011)

El algoritmo de construcción puede continuar indefinidamente, y la estructura resultante se puede usar en una amplia variedad de aplicaciones de la enseñanza de matemáticas, desde en simples procedimientos de construcción hasta en trigonometría.

\section{Conocimiento matemático ético}

El conocimiento matemático ético está relacionado con las cuentas, descripciones y análisis de las ideas, conceptos, procedimientos y prácticas matemáticas ex- 
presados en términos de las categorías que se consideran significativos y apropiados por la comunidad de observadores científicos. Este tipo de conocimiento debe ser preciso, lógico, completo, replicable e independiente de los observadores externos, que tienen una visión desde fuera, exterior (Rosa y Orey, 2017). La figura 7 muestra un ejemplo de conocimiento matemático ético.

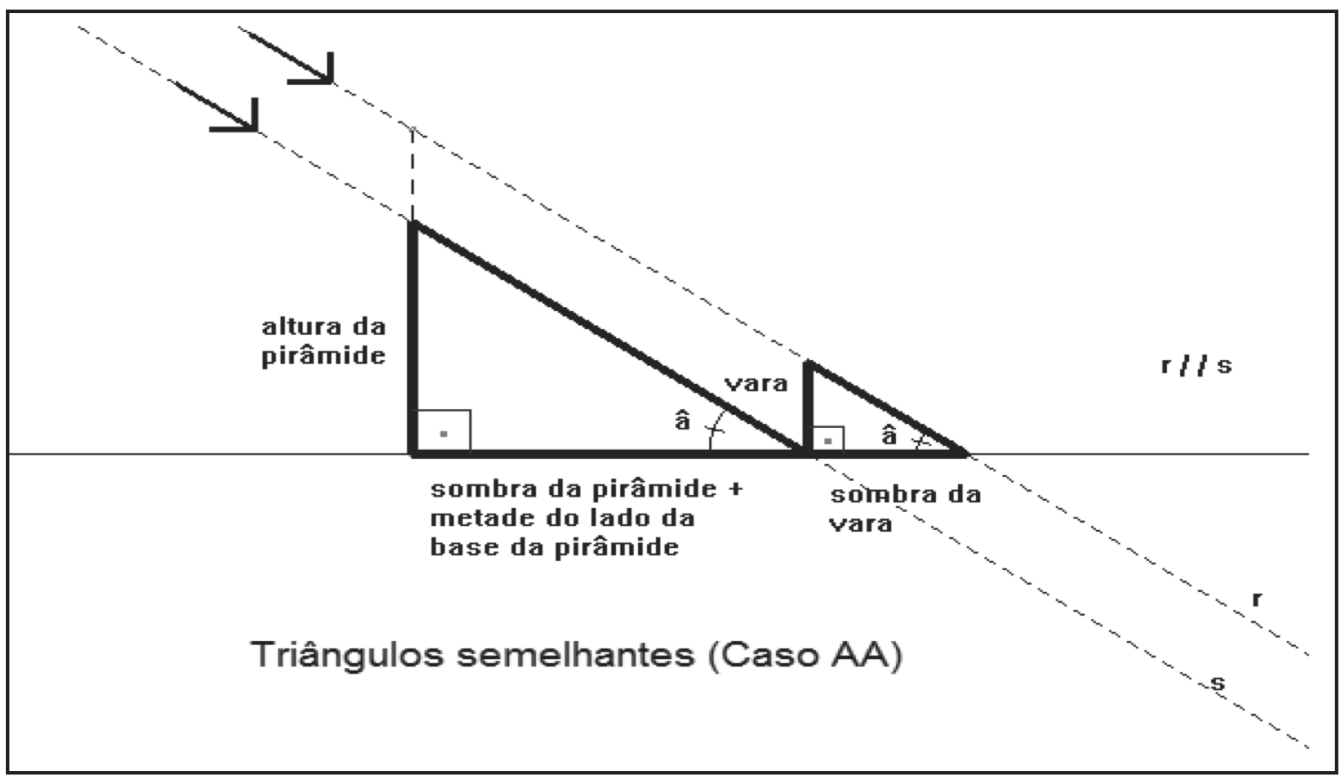

Figura 7. Conocimiento matemático émico.

Fuente: Rosa y Orey (2017)

Para Rosa y Orey (2017), la validación del conocimiento ético es una cuestión de análisis lógico y empírico, en particular, de que la construcción cumple con los estándares de integralidad y consistencia lógica.

\section{Un ejemplo de conocimiento matemático ético}

La modelación de una pared en una escuela de Ouro Preto, Minas Gerais, Brasil (Rosa y Orey, 2010) fue realizada para comprobar si se podía demostrar que la forma geométrica mostrada era una serie de curvas exponenciales, parábolas o catenarias.

Lo que sucedió fue algo totalmente sorprendente, y al final, los resultados, por importantes que sean, fueron eclipsados por la oportunidad de discutir y debatir acerca de las curvas exponenciales, parabólicas y catenarias que se dio entre los profesores y los estudiantes. La figura 8 muestra la pared de la escuela, las curvas y el etnomodelo gráfico (Rosa y Orey, 2010). 


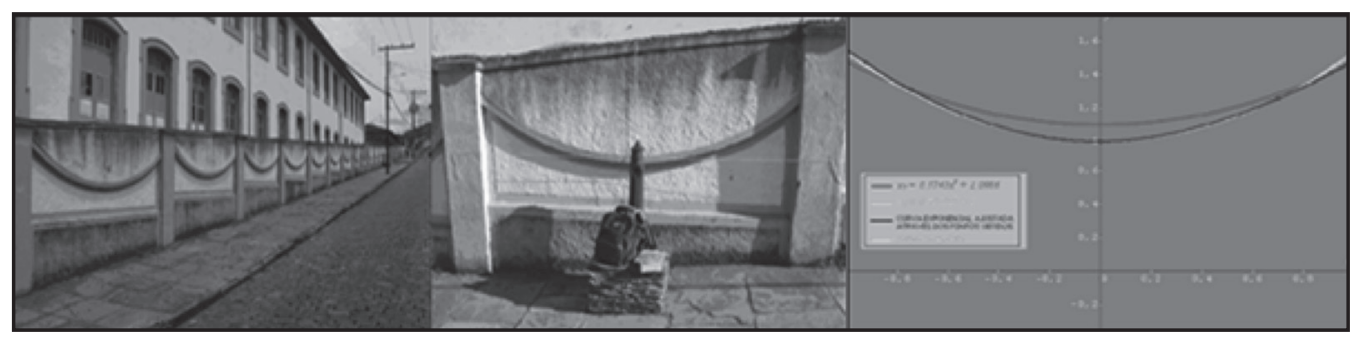

Figura 8. Pared de la escuela, las curvas y el etnomodelo gráfico.

Fuente: Rosa y Orey (2010)

Después de examinar los datos recogidos al medir varias curvas en la pared y tratar de adaptarlos a las funciones exponencial, cuadrática y catenaria, a través de modelos matemáticos, llegamos a la conclusión de que las curvas de la pared se aproximan a una curva catenaria.

\section{Etnomodelos}

La elaboración de etnomodelos que representan estos sistemas son representaciones que ayudan a los miembros de diferentes grupos a entender y apropiarse de la realidad mediante el uso de pequeñas unidades de información, denominadas etnomodelos, émicos o éticos, que vinculan su patrimonio cultural al desarrollo de sus prácticas matemáticas (Rosa y Orey, 2017).

Por ejemplo, Cortes (2017) afirma que los etnomodelos émicos están fundamentados en las informaciones matemáticas que son importantes para los miembros de la propia comunidad, pues representan su pensamiento matemático, y buscan traducir su forma de pensar matemáticamente utilizando ejemplos que se encuentran en su propia realidad.

Por otro lado, Cortes (2017) sostiene que los etnomodelos éticos se basan en la visión de los observadores externos acerca de la realidad que se está modelando, pues representan cómo los modeladores piensan que el mundo funciona a través de sistemas tomados de la realidad.

\section{La perspectiva dialógica (émica-ética)}

La perspectiva dialógica presenta un dinamismo cultural entre los conocimientos émico y ético. Por ejemplo, la perspectiva ética juega un papel importante en la investigación en etnomodelación, sin embargo, la perspectiva émica debe tenerse en cuenta en este proceso también, ya que las características émicas acentúan la cuestión de lo que debe incluir un modelo basado en agentes para servir a objetivos prácticos en la investigación de etnomodelos (Rosa y Orey, 2017).

Así, para Cortes (2017), el abordaje dialógico puede considerarse como la aceleración e intensificación de la interacción e integración entre los miembros de grupos 
culturales distintos. La interrelación entre la cultura local y las ideas matemáticas puede ser mutuamente reforzada por la utilización de actividades matemáticas culturalmente relevantes.

Estas actividades ayudan a los estudiantes a percibir la relevancia de las matemáticas y auxilian a los profesores a utilizar esta conexión para enseñar más matemáticas mediante el uso de las prácticas matemáticas a través de las etnomatemáticas y de los artefactos culturales.

\section{Etnomodelos dialógicos}

Las ideas y procedimientos matemáticos son éticos si pueden ser comparados entre diferentes culturas que utilizan definiciones, categorías y métricas comunes, mientras que el énfasis del análisis local de estos aspectos es émico si las ideas y procedimientos matemáticos $y$, también las prácticas, son desarrollados exclusivamente por los miembros de un subconjunto de culturas que tienen sus raíces en las diversas formas en que las actividades éticas se llevan a cabo en un entorno culturalmente específico (Cortes, 2017).

Desde ese enfoque, la perspectiva ética reconoce que el conocimiento de los miembros de cualquier grupo cultural determinado no tiene prioridad sobre sus contendientes afirmaciones émicas, y viceversa. Por lo tanto, hay una necesidad de la realización de "actos de traducción" entre las perspectivas émica y ética (Eglash et al., 2006).

En ese sentido, Rosa y Orey (2010) sostienen que el conocimiento matemático de los miembros de diferentes grupos culturales se combina con el sistema de conocimiento matemático occidental para resultar en una perspectiva dialógica en educación matemática por medio del dinamismo cultural.

\section{Un ejemplo de conocimiento matemático dialógico}

La base trípode del tipi de los indígenas sioux de los Estados Unidos parece ser perfectamente adaptada para el duro ambiente en el que se utiliza y tiene la ventaja de proporcionar una estructura estable, pues resiste los vientos y el clima extremadamente variable que impera en aquella región (Orey, 2000). La figura 8 muestra el tipi, su base trípode y sus matematizaciones.
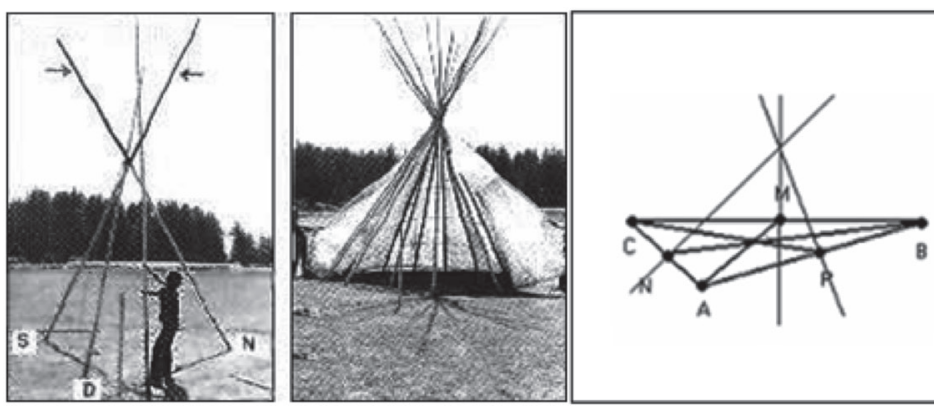

Figura 9. tipi, su base trípode y sus matematizaciones.

Fuente: Orey (2000) 
En ese contexto, Orey (2000) afirma que los habitantes determinaban el centro de la base circular del tipi usando la idea del triángulo existente formado por el trípode. El centro del tipi tiene un poder y santidad definido, es algo más que solo necesidad o estética, y pasa a ser el centro de la casa sioux.

\section{Un currículo para la etnomodelación}

La utilización de la matematización que está presente en la cotidianeidad de los miembros de grupos culturales tiene por objetivo la ampliación y el perfeccionamiento del conocimiento matemático, pues conduce al fortalecimiento de la identidad cultural de los individuos, como seres autónomos y capaces (Rosa y Orey, 2012).

La etnomodelación es una de las posibles estrategias para la enseñanza que hará posible aproximar y relacionar los saberes escolares (ético) y el día a día (émico) por medio del dinamismo cultural, pues puede ser considerada como el estudio de los fenómenos matemáticos dentro de una cultura y se trata de una construcción social culturalmente delimitada (Cortes, 2017).

Así, Rosa y Orey (2017) afirman que los desarrolladores de currículo han hecho caso omiso a las perspectivas émicas en las actividades curriculares escolares y que, tal vez, esta sea una de las principales razones del fracaso de muchos sistemas educativos en el mundo.

Siendo así, se hace necesario incorporar elementos matemáticos ancestrales de la comunidad local al currículo escolar porque la etnomodelación es una metodología científica que tiene como característica la organización de las estrategias de enseñanza en una vertiente pedagógica cuyo objetivo es la reorganización del currículo matemático, pues pretende atender las demandas del mundo moderno (Rosa y Orey, 2017).

Una perspectiva dialógica (émica-ética) incluye el reconocimiento de otras epistemologías y de la naturaleza holística e integrada del conocimiento matemático de los miembros de diversos grupos culturales que se encuentran en muchos centros urbanos (Rosa y Orey, 2012). Sin embargo, Rosa (2010) afirma que la organización de situaciones didácticas desde una perspectiva etnomatemática utiliza la modelación como uno de los posibles caminos para concretizarse un trabajo centrado en una perspectiva etnomatemática en clase.

\section{Consideraciones finales}

Con la creciente preocupación por la incorporación de la perspectiva etnomatemática a los currículos de matemáticas, existe la necesidad de desarrollar una práctica etnomatemática dirigida a la acción pedagógica. Por lo tanto, un objetivo alternativo para la investigación debe ser la adquisición de conocimiento tanto émico como ético en etnomodelación.

El conocimiento émico es una valiosa fuente de inspiración para la hipótesis ética y viceversa. El conocimiento ético es esencial para la comparación intercultural, el 
componente esencial de la etnología. Debido a que es una construcción social vinculada culturalmente, y una parte esencial de la etnomatemática, la etnomodelación se define como el estudio de los fenómenos matemáticos dentro de una cultura. Sin olvidar que éstos están permeados o que están íntimamente relacionados con la cosmovisión de los pueblos.

Así mismo, hay que hacer un llamamiento a los programas de los diferentes grados académicos para que incorporen en sus programas la reflexión sobre la etnomatemática; $y$, por otra parte, se hace necesario que los investigadores acompañen de manera más cercana y comprometida a los docentes en este proceso de recuperación y conservación del patrimonio matemático autóctono de las comunidades locales.

Desde nuestro punto de vista, el programa etnomatemáticas no puede preocuparse solamente de la vertiente antropológica y etnográfica de la descripción de diferentes pensamientos matemáticos, pues también debe asumir una perspectiva dirigida a los aspectos pedagógicos del currículo escolar. Así, la etnomodelación debe proporcionar a los estudiantes una acción pedagógica que conecte las prácticas matemáticas locales a los conocimientos de la matemática académica.

Es importante que los individuos desarrollen sus propias prácticas matemáticas; sin embargo, es fundamental que también tengan una comprensión de la institución sociopedagógica de la matemática académica por medio de acciones pedagógicas curriculares que fomenten la conexión de las prácticas matemáticas presentes en la comunidad con las prácticas matemáticas enseñadas en las escuelas, en una reinterpretación del currículo matemático escolar.

En ese contexto, de acuerdo con D'Ambrosio (2000), los individuos esperan, en esta fase de la evolución de nuestra especie, que toda la arrogancia, envidia y gran poder ceda lugar al respeto por los diversos pueblos que, en solidaridad, contribuirán a la preservación del patrimonio común.

\section{Referencias}

Babbitt, L., Lyles, D., \& Eglash, R. (2011). From ethnomathematics ti ethnocomputing: indigenous algorithms in traditional context \& contemporary simulation. In Alternative forms of knowing (in) mathematics: celebrations of diversity of mathematical practices (pp. 215-219). Rotterdam, The Netherlands: Sense Publishers.

Bassanezi, R. C. (2002). Ensino-aprendizagem com modelagem matemática. São Paulo, SP: Editora Contexto.

Cortes, D. P. O. (2017). Re-significando os conceitos de função: um estudo misto para entender as contribuiçôes da abordagem dialógica da etnomodelagem. Dissertação (Mestrado Profissional em Educação Matemática). Instituto de Ciências Exatas e Biológicas - ICEB. Departamento de Educaçáo Matemática - DEEMA. Ouro Preto, MG: UFOP. 2017. 
D’Ambrosio, U. (1990). Etnomatemática. São Paulo, SP: Editora Ática.

D'Ambrosio, U. (2000). Etnomatemática e modelagem. Anais do Primeiro Congresso Nacional de Etnomatemática - CBEm1 (pp. 142-143). São Paulo, SP: FEUSP.

Eglash, R. (2002). African fractals: modern computing and indigenous design. New Brunswick, NJ: Rutgers University Press.

Eglash, R. (2006). Culturally situated designed tools: ethnocomputing from field site to classroom. American Anthropologist, 108(2), 347-362.

Gerdes, P. (1993). Geometria Sona, reflexōes sobre uma tradição de desenhos em povos, cultura, matemática, educaçâo. Maputo, Moçambique: Instituto Superior Pedagógico.

Knijnik, G. (1993). O saber popular e o saber acadêmico na luta pela terra. A Educação Matemática em Revista, 1(1), 28-42.

Lewis, S. T. (2018). Theorizing teaching practices in mathematical modeling contexts through the examination of teacher scaffolding. Graduate School. Doctorate Dissertation. Columbus, $\mathrm{OH}$ : The Ohio State University.

Monteiro, A. (2004). A etnomatemática em cenários de escolarização: alguns elementos de reflexão. In G. Knijnik, F. Wanderer, \& C. Oliveira (Eds.), Etnomatemática: currículo e formação de professores (pp. 432-446). Santa Cruz do Sul, RS: EDUNISC.

Monteiro, A., \& Pompeu Jr., G. P. (2003). A matemática e os temas transversais. São Paulo, SP: Editora Moderna.

Orey, D. C. (2000). The ethnomathematics of Sioux tipi and cone. In Selin, H. (Ed.), Mathematics across cultures: the history of non-western mathematics (pp. 239-253). Norwell, Netherlands: Kluwer Academic Publishers.

Rios, D. P. (2000). Primero etnogeometría para seguir con etnomatemática. In Domite, M. C. (Ed.), Anais do Primeiro Congresso Brasileiro de Etnomatemática CBEm-1 (pp. 367-375). São Paulo, SP: FE-USP.

Rosa, M. (2010). A mixed-method study to understand the perceptions of high school leaders about English language Learners (ELLs): The case of mathematics. College of Education. Tese de Doutorado Educação - Liderança Educacional. Sacramento, CA: California State University - CSUS.

Rosa, M., \& Orey, D. C. (2003). Vinho e queijo: etnomatemática e modelagem. BOLEMA, 16(20), 1-16.

Rosa, M., \& Orey, D. C. (2006). Abordagens atuais do programa etnomatemática: delinenando-se um caminho para a ação pedagógica. BOLEMA, 19(26), 19-48.

Rosa, M., \& Orey, D. C. (2010). Ethnomodeling as a pedagogical tool for the ethnomathematics program. Revista Latinoamericana de Etnomatemática, 3(2), p. 14- 23.

Rosa, M., \& Orey, D. C. (2012). O campo de pesquisa em etnomodelagem: as abordagens êmica, ética e dialética. Educação e Pesquisa, 38(4), 865-879.

Rosa, M., \& Orey, D. C. (2017). Etnomodelagem: a arte de traduzir práticas matemáticas locais. São Paulo, SP: Editora Livraria da Física. 